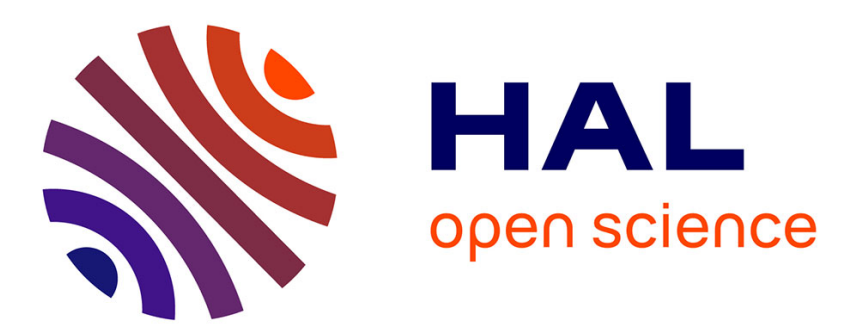

\title{
On the effective stiffness of plates made of hyperelastic materials with initial stresses
}

\author{
H. Altenbach, V.A. Eremeyev
}

\section{To cite this version:}

H. Altenbach, V.A. Eremeyev. On the effective stiffness of plates made of hyperelastic materials with initial stresses. International Journal of Non-Linear Mechanics, 2010, 45 (10), pp.976. 10.1016/j.ijnonlinmec.2010.04.007 . hal-00698849

\section{HAL Id: hal-00698849 \\ https://hal.science/hal-00698849}

Submitted on 18 May 2012

HAL is a multi-disciplinary open access archive for the deposit and dissemination of scientific research documents, whether they are published or not. The documents may come from teaching and research institutions in France or abroad, or from public or private research centers.
L'archive ouverte pluridisciplinaire HAL, est destinée au dépôt et à la diffusion de documents scientifiques de niveau recherche, publiés ou non, émanant des établissements d'enseignement et de recherche français ou étrangers, des laboratoires publics ou privés. 
On the effective stiffness of plates made of hyperelastic materials with initial stresses

H. Altenbach, V.A. Eremeyev

PII:

S0020-7462(10)00073-9

DOI: doi:10.1016/j.ijnonlinmec.2010.04.007

Reference: NLM 1723

To appear in: International Journal of Non-

www.elsevier.com/locate/nlm Linear Mechanics

Received date: 31 October 2008

Revised date: 14 March 2010

Accepted date: 13 April 2010

Cite this article as: H. Altenbach and V.A. Eremeyev, On the effective stiffness of plates made of hyperelastic materials with initial stresses, International Journal of Non-Linear Mechanics, doi:10.1016/j.jinonlinmec.2010.04.007

This is a PDF file of an unedited manuscript that has been accepted for publication. As a service to our customers we are providing this early version of the manuscript. The manuscript will undergo copyediting, typesetting, and review of the resulting galley proof before it is published in its final citable form. Please note that during the production process errors may be discovered which could affect the content, and all legal disclaimers that apply to the journal pertain. 


\title{
On the Effective Stiffness of Plates made of Hyperelastic Materials with Initial Stresses
}

\author{
H. Altenbach ${ }^{\mathrm{a}, *}$, V.A. Eremeyev ${ }^{\mathrm{b}}$ \\ ${ }^{a}$ Martin-Luther-Universität Halle-Wittenberg, D - 06099 Halle (Saale), Germany \\ ${ }^{\mathrm{b}}$ South Scientific Center of RASci \&3 South Federal University, \\ Milchakova str. 8a, 344090 Rostov on Don, Russia
}

\begin{abstract}
Within the framework of the direct approach to the plate theory we consider the infinitesimal deformations of a plate made of hyperelastic materials taking into account the non-homogeneously distributed initial stresses. Here we consider the plate as a material surface with 5 degrees of freedom ( 3 translations and 2 rotations). Starting from the equations of the non-linear elastic body and describing the small deformations superposed on the finite deformation we present the twodimensional constitutive equations for a plate. The influence of initial stresses in the bulk material on the plate behavior is considered.
\end{abstract}

Key words: Nonlinear elasticity, Prestressed material, Plate theory, Initial stresses, Foam

\section{Introduction}

Thin-walled engineering structures made of hyperelastic porous materials, such as polymer foams, have different applications in the last decades [14]. A polymer foam is a cellular structure consisting of a solid polymer, for example polyurethane, etc., containing a large volume fraction of gas-filled pores. There are two types of foams. One is the closed-cell foam, while the second one is the open-cell foam. The defining characteristics of foams is the

\footnotetext{
* Corresponding author.

Email addresses: holm.altenbach@iw.uni-halle.de (H. Altenbach), eremeyev.victor@gmail.com (V.A. Eremeyev).
} 
very high porosity: typically well over $80 \%, 90 \%$ and even $98 \%$ of the volume consists of void spaces. The porosity and the topology of a foam determine the other properties, such as for example, Young's modulus, etc.

Polymer foams may demonstrate very large elastic strains. Hence such foams may be considered as a non-linear hyperelastic material. Different models allowing the description of large hyperelastic deformations of foams are proposed in the literature $[3,4]$. The existing models of foams may be classified as follows. The first type of models bases on the detailed considerations of the foam cell deformation taking into account the cell structure, the properties of cell walls and struts, the pressure change in the closed cells, etc., see [3-9] among others. The famous Kelvin model of foam belongs to this type. On the other hand the computational efforts may be significant and there is hard to establish experimentally the real material properties of cells. The second class of models use the description of a foam as the continuum media. Within the framework of this type models, one takes into account the structure of foam cells, the solid material and gas properties and other parameters in the constitutive equations at whole. The Ogden's material model is applied for the finite deformations of hyperelastic foams, see [4] and [10-17]. Both types of models of hyperelastic foams have advantages and disadvantages. Further we apply the second approach using the Ogden's material model of hyperelastic material for the moderate large strain and for the low level of stress field.

There are many plate-like engineering structures made of foams, for example sandwich plates with a core made of foam, laminates, etc., see $[3,4]$ for details. Within the framework of the theory of plates and shells [18-23] the theory of elastic plates with nonhomogeneous distribution of the porosity (functionally graded plates) is developed in [24] while the theory of viscoelastic plates is presented in $[25,26]$, see also [27].

For the structures under consideration the initial stresses may influence on the plate behavior. The mechanics of the prestressed three-dimensional solids is developed in numerous papers and books, see [13,28-32] among others. The aim of this paper is to extend the results of [24] to plates made of material with internal stresses using the theory of small deformations superposed on finite deformation presented in the mentioned works. Let us note that the Kirchhoff-Love linear theory of shells made of prestressed material was earlier developed in a number of papers, see for example [30,33-36]. Here we consider the theory of plates taking into account the transverse shear deformations like in the theories proposed by Reissner [37,38] and Mindlin [39], see also the review [40].

The paper is organized as follows. In Sect. 1 we recall the basic equation of the three-dimensional theory of nonlinear elasticity. We present here the governing equations describing the infinitesimal deformations of a prestressed 
body. Further in Sect. 2 we consider the linearized equilibrium equations of a three-dimensional plate-like body with the initial stresses depending on the thickness coordinate. In Sect. 3 we give the statement of the two-dimensional boundary-value problems for the linear plate theory.

\section{Basic equations of 3D non-linear elasticity}

Following [13,29-32] in this section we present the general equations governing small (incremental) deformations superimposed on a finite homogeneous deformation in an compressible elastic material. The Eulerian equilibrium equations of the non-linear body are given by the relations

$$
\operatorname{div} \boldsymbol{\tau}+\rho \boldsymbol{f}=\mathbf{0}, \quad \boldsymbol{\tau}=J^{-1} \mathbf{F} \cdot \mathbf{S}, \quad \mathbf{S}=\frac{\partial W}{\partial \mathbf{F}},
$$

where div is the divergence operator in the actual configuration $\chi, \boldsymbol{\tau}$ the Cauchy stress tensor, $\mathbf{S}$ the $1^{\text {st }}$ Piola-Kirchhoff stress tensor, $\rho$ the material density in the actual configuration, $\boldsymbol{f}$ the body force vector per unit mass, $W$ the strain-energy function (per unit volume), $J=\operatorname{det} \mathbf{F}$, and $\mathbf{F}$ is the deformation gradient defined as in [13]. Note that here we use the notation $\mathbf{A} \cdot \boldsymbol{a}$ and $\mathbf{A} \cdot \mathbf{B}$ for the second-order tensors $\mathbf{A}$ and $\mathbf{B}$, and a vector $\boldsymbol{a}$ instead of the alternative way $\mathbf{A} \boldsymbol{a}$, and $\mathbf{A B}$, respectively. Further we assume the isotropic behavior of the material, so we use the constitutive equation in the following form:

$$
W=W\left(I_{1}, I_{2}, I_{3}\right)
$$

where $I_{1}, I_{2}, I_{3}$ are the principal invariants of the left Cauchy-Green deformation tensor $\mathbf{b}=\mathbf{F} \cdot \mathbf{F}^{\mathrm{T}}$ or the right Cauchy-Green deformation tensor $\mathbf{c}=\mathbf{F}^{\mathrm{T}} \cdot \mathbf{F}$, defined by

$$
\begin{aligned}
I_{1}=\operatorname{tr} \mathbf{b}=\operatorname{tr} \mathbf{c} & =\lambda_{1}^{2}+\lambda_{2}^{2}+\lambda_{3}^{2}, \\
I_{2}=\frac{1}{2}\left[\operatorname{tr}^{2} \mathbf{b}-\operatorname{tr} \mathbf{b}^{2}\right]=\frac{1}{2}\left[\operatorname{tr}^{2} \mathbf{c}-\operatorname{tr} \mathbf{c}^{2}\right] & =\lambda_{1}^{2} \lambda_{2}^{2}+\lambda_{2}^{2} \lambda_{3}^{2}+\lambda_{1}^{2} \lambda_{3}^{2}, \\
I_{3}=\operatorname{det} \mathbf{b}=\operatorname{det} \mathbf{c} & =\lambda_{1}^{2} \lambda_{2}^{2} \lambda_{3}^{2} .
\end{aligned}
$$

Here $\lambda_{1}, \lambda_{2}, \lambda_{3}$ are the principal stretches, tr denotes the trace of a secondorder tensor, and $(\ldots)^{\mathrm{T}}$ denotes transposed. $\lambda_{1}, \lambda_{2}, \lambda_{3}$ may be also considered as the arguments of the strain function $W$ :

$$
W=W\left(\lambda_{1}, \lambda_{2}, \lambda_{3}\right)
$$


For the isotropic material $\mathbf{S}$ and $\boldsymbol{\tau}$ are given by the relations

$$
\begin{aligned}
& \mathbf{S}=2 \frac{\partial W}{\partial \mathbf{c}} \cdot \mathbf{F}^{\mathrm{T}}=\left(f_{0} \mathbf{c}^{-1}+f_{1} \mathbf{I}+f_{2} \mathbf{c}\right) \cdot \mathbf{F}^{\mathrm{T}}, \\
& \boldsymbol{\tau}=J^{-1} \mathbf{F} \cdot \mathbf{S}=f_{0} \mathbf{I}+f_{1} \mathbf{b}+f_{2} \mathbf{b}^{2}
\end{aligned}
$$

where $\mathbf{I}$ is the unit second-order tensor, $f_{0}, f_{1}, f_{2}$ are functions which may be expressed as combinations of the partial derivatives of $W$ with respect to $I_{i}$ or $\lambda_{i}$, see $[13,29,30]$ for details.

For the description of the non-linear behavior of polymeric foams the following constitutive equation is widely used [4]

$$
\begin{aligned}
W & =\sum_{i=1}^{N} \frac{2 \mu_{i}}{\alpha_{i}^{2}}\left[\operatorname{tr} \mathbf{b}^{\alpha_{i} / 2}-3+\frac{1}{\beta_{i}}\left(\operatorname{det} \mathbf{F}^{-\alpha_{i} \beta_{i}}-1\right)\right] \\
& =\sum_{i=1}^{N} \frac{2 \mu_{i}}{\alpha_{i}^{2}}\left[\lambda_{1}^{\alpha_{i}}+\lambda_{2}^{\alpha_{i}}+\lambda_{3}^{\alpha_{i}}-3+\frac{1}{\beta_{i}}\left(J^{-\alpha_{i} \beta_{i}}-1\right)\right],
\end{aligned}
$$

where $\mu_{i}, \alpha_{i}, \beta_{i}$ are the elastic moduli $(i=1 \ldots N)$. Here

$$
\mu=\sum_{i=1}^{N} \mu_{i}
$$

$\mu$ denotes the initial shear modulus, while the initial bulk modulus $k$ is given by

$$
k=\sum_{i=1}^{N} 2 \mu_{i}\left(\beta_{i}+\frac{1}{3}\right) .
$$

The model (4) was originally proposed by Ogden [11,12], see also $[4,16,10,17,15]$ among others, where Ogden's model is used. For some special choice of the values $\mu_{i}, \alpha_{i}, \beta_{i}$ and $N$, Ogden's strain function $W$ reduces to various others models applied in the nonlinear elasticity (neo-Hookean, Varga, Mooney-Rivlin, Blatz-Ko constitutive equations, etc.).

Using the identity $\operatorname{Div}\left(J^{-1} \mathbf{F}\right)=\mathbf{0}$ we transform Eqs (1) to the Lagrangian form

$$
\operatorname{Div} \mathbf{S}+\rho_{0} \boldsymbol{f}=\mathbf{0},
$$

where Div is the divergence operator in the reference configuration, and $\rho_{0}$ the density in this configuration.

Let us consider the equilibrium equations of a prestressed body. In other words, we introduce the small deformations superposed on the finite deformation. Let $\boldsymbol{x}$ be the known position vector in the actual configuration $\chi$ while $\boldsymbol{x}+\boldsymbol{w}$ is the position vector in another actual configuration $\chi^{\star}$ which differs from $\chi$ by the infinitesimal vector $\boldsymbol{w}$. 
The linearization of Eq. (5) results in [13,29-32]

$$
\operatorname{Div} \mathbf{S}^{\star}+\rho_{0} \boldsymbol{f}^{\star}=\mathbf{0},
$$

where

$$
\mathbf{S}^{\star}=\frac{\partial^{2} W}{\partial \mathbf{F} \partial \mathbf{F}} \cdot \mathbf{F}^{\star \mathrm{T}}, \quad \mathbf{F}^{\star}=\operatorname{Grad} \boldsymbol{w}
$$

and Grad is the gradient operators in the reference configuration, $f^{\star}$ is the small additional body force acting in the actual configuration $\chi^{\star}$, and $\cdot \cdot$ is the double dot (inner) product.

The Lagrangian linearized equilibrium equation (6) may be transform to the Eulerian form

$$
\operatorname{div} \boldsymbol{\Theta}+\rho \boldsymbol{f}^{\star}=\mathbf{0},
$$

where $\boldsymbol{\Theta}$ is the linearized stress tensor given by formulas $[29,31]$

$$
\Theta=J^{-1} \mathbf{F} \cdot \mathbf{S}^{\star}
$$

For example, let us consider the derivation procedure of $\mathbf{S}^{\star}$ and $\boldsymbol{\Theta}$ for the special case of (4) with $N=1, \alpha_{1}=2, \mu_{1}=\mu, \beta_{1}=\beta$. Here we have the constitutive relations

$$
\begin{gathered}
W=\frac{\mu}{2}\left[\operatorname{tr} \mathbf{c}-3+\frac{1}{\beta}\left(J^{-2 \beta}-1\right)\right], \\
\mathbf{S}=\mu \mathbf{F}^{T}-\mu J^{-2 \beta} \mathbf{F}^{-1}, \quad \boldsymbol{\tau}=\mu J^{-1} \mathbf{b}-\mu J^{-2 \beta-1} \mathbf{I} .
\end{gathered}
$$

Using the latter relations and the formula $J^{\star}=J \operatorname{div} \boldsymbol{w}$ we established the following relations for $\mathbf{S}^{\star}$ and $\Theta$

$$
\begin{gathered}
\mathbf{S}^{\star}=\mu \mathbf{F}^{\star T}+\mu J^{-2 \beta} \mathbf{F}^{-1} \cdot \mathbf{F}^{\star} \cdot \mathbf{F}^{-1}+2 \mu \beta J^{-2 \beta}(\operatorname{div} \boldsymbol{w}) \mathbf{F}^{-1} \\
\boldsymbol{\Theta}=\mu J^{-1} \mathbf{F} \cdot \mathbf{L}^{T} \cdot \mathbf{F}^{T}+\mu J^{-2 \beta-1} \mathbf{L}+2 \mu \beta J^{-2 \beta-1}(\operatorname{div} \boldsymbol{w}) \mathbf{I} \\
\mathbf{L}=\mathbf{F}^{\star} \cdot \mathbf{F}^{-1} \equiv \operatorname{grad} \boldsymbol{w} .
\end{gathered}
$$

Here grad is the gradient operators in the actual $(\chi)$ configuration. Note that for the case $\mathbf{F}=\mathbf{I}$ Eqs (9) reduce to the Hooke's law

$$
\mathbf{S}^{\star}=\boldsymbol{\Theta}=2 \mu \varepsilon+2 \beta \mu \mathbf{I t r} \boldsymbol{\varepsilon}, \quad \boldsymbol{\varepsilon}=\frac{1}{2}\left(\mathbf{L}+\mathbf{L}^{T}\right) .
$$

The equilibrium equations (7) or (6) describe the prestressed solid deformable body as a result of infinitesimal deformations. From this point of view one may consider the relations $\Theta=\boldsymbol{\Theta}(\mathbf{L})$ or $\mathbf{S}^{\star}=\mathbf{S}^{\star}\left(\mathbf{F}^{\star}\right)$ as the constitutive relations of the prestressed body. Of course, $\boldsymbol{\Theta}$ and $\mathbf{S}^{\star}$ depend also on the 


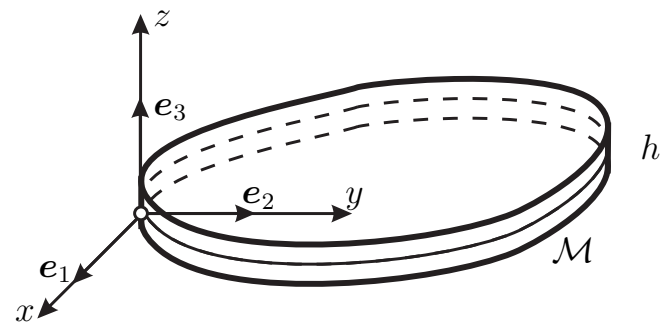

Fig. 1. 3D plate-like body

initial deformation gradient $\mathbf{F}$. Let us note that in the general case the tensors $\boldsymbol{\Theta}$ and $\mathbf{S}^{\star}$ are non-symmetric. That means that these "constitutive equations" differ from the Hooke's law of the linear elasticity. On the other hand such symmetry may be established for the special cases of initial strain or stress, see [29]. This model of prestressed body includes also the induced anisotropy effects because the tensor of instantaneous elastic moduli may corresponded to an anisotropic body.

\section{Equilibrium equations for prestressed plate-like body}

Let us consider the prestressed plate-like body which occupies the volume $V=\left\{(x, y, z):(x, y) \in M \subset \mathbb{R}^{2}, z \in[-h / 2, h / 2]\right\}$, where $h$ is the platelike body thickness, Fig. 1. We assume that the body is nonhomogeneous in $z$-direction, i.e. $W$ depends on $z$

$$
W=W\left(I_{1}, I_{2}, I_{3}, z\right)
$$

and the initial stretches depend only on $z$ :

$$
\lambda_{1}=\lambda_{2}=\lambda(z), \quad \lambda_{3}=\lambda_{3}(z) .
$$

The principal Cauchy stresses $\tau_{1}, \tau_{2}, \tau_{3}$ can be calculated according to the formula $[13,31]$

$$
\tau_{i}=J^{-1} \lambda_{i} \frac{\partial W}{\partial \lambda_{i}} \quad(i=1,2,3),
$$

which also depend only on $z$. If the body forces and the surface loads are consistent with these assumptions then the initial state describes the axialsymmetric deformations of the nonhomogeneous plate-like body.

Let us consider the constitutive equation $\mathbf{\Theta}=\mathbf{\Theta}(\mathbf{L})$ or $\mathbf{S}^{\star}=\mathbf{S}^{\star}\left(\mathbf{F}^{\star}\right)$ for such initial state of a plate-like body. The tensors $\mathbf{b}$ and $\mathbf{c}$ are given by

$$
\mathbf{b}=\mathbf{c}=\lambda^{2}(z)\left(\boldsymbol{e}_{1} \boldsymbol{e}_{1}+\boldsymbol{e}_{2} \boldsymbol{e}_{2}\right)+\lambda_{3}^{2}(z) \boldsymbol{e}_{3} \boldsymbol{e}_{3}
$$


where $\boldsymbol{e}_{1}, \boldsymbol{e}_{2}, \boldsymbol{e}_{3}$ are the Cartesian basis vectors. For the isotropic material behavior from Eq. (3) it follows that $\boldsymbol{\tau}$ is reduced to the relation

$$
\boldsymbol{\tau}=\tau(z)\left(\boldsymbol{e}_{1} \boldsymbol{e}_{1}+\boldsymbol{e}_{2} \boldsymbol{e}_{2}\right)+\tau_{3}(z) \boldsymbol{e}_{3} \boldsymbol{e}_{3},
$$

where $\tau=\tau_{1}=\tau_{2}$.

Using the relation $\mathbf{c}^{\star}=2 \mathbf{F}^{\mathrm{T}} \cdot \boldsymbol{\varepsilon} \cdot \mathbf{F}$ with $2 \boldsymbol{\varepsilon}=\mathbf{L}+\mathbf{L}^{\mathrm{T}}$ we obtain

$$
\begin{aligned}
\mathbf{S}^{\star} & =2\left(\frac{\partial^{2} W}{\partial \mathbf{c}^{2}} \cdots \mathbf{c}^{\star T}\right) \cdot \mathbf{F}^{\mathrm{T}}+2 \frac{\partial W}{\partial \mathbf{c}} \cdot \mathbf{F}^{\star \mathrm{T}} \\
& =4\left[\frac{\partial^{2} W}{\partial \mathbf{c}^{2}} \cdots\left(\mathbf{F}^{\mathrm{T}} \cdot \boldsymbol{\varepsilon} \cdot \mathbf{F}\right)\right] \cdot \mathbf{F}^{\mathrm{T}}+\mathbf{S} \cdot \mathbf{L}, \\
\boldsymbol{\Theta} & =\mathcal{A} \cdot \boldsymbol{\varepsilon}+\boldsymbol{\tau} \cdot \mathbf{L},
\end{aligned}
$$

where $\mathcal{A}$ is the $4^{\text {th }}$-order tensor of instantaneous elastic moduli defined in Cartesian base as

$$
\mathcal{A} \equiv \mathcal{A}_{i m s t} \boldsymbol{e}_{i} \boldsymbol{e}_{m} \boldsymbol{e}_{s} \boldsymbol{e}_{t}=F_{i a} F_{s d} F_{t c} F_{m b} \frac{\partial^{2} W}{\partial c_{a b} \partial c_{c d}} \boldsymbol{e}_{i} \boldsymbol{e}_{m} \boldsymbol{e}_{s} \boldsymbol{e}_{t}
$$

It is easy to see that $\mathcal{A}$ has pair-wise symmetry properties such as

$$
\mathcal{A}_{\text {imst }}=\mathcal{A}_{\text {stim }}=\mathcal{A}_{\text {mist }} \text {. }
$$

As an example let us consider the homogeneous initial hydrostatically stressed state. Here we have the relations

$$
\begin{gathered}
\mathbf{F}=\lambda \mathbf{I}, \quad \mathbf{b}=\mathbf{c}=\lambda^{2} \mathbf{I}, \quad \boldsymbol{\tau}=-p \mathbf{I}, \quad-p=f_{0}+f_{1} \lambda^{2}+f_{2} \lambda^{4}, \\
I_{1}=3 \lambda^{2}, \quad I_{2}=3 \lambda^{4}, \quad J=\lambda^{3} .
\end{gathered}
$$

For the general constitutive equations it may be proved that the expression of $\boldsymbol{\Theta}$ reduces to the Hooke's law with the Làme constant depending on $p$, see [29]. For the special case (8) the pressure $p$ is given by the relation

$$
p=\mu\left(\frac{1}{\lambda^{6 \beta+3}}-\frac{1}{\lambda}\right) \text {. }
$$

Substituting (11) into (9) we obtain

$$
\boldsymbol{\Theta}=\mu\left(\frac{1}{\lambda}+\frac{1}{\lambda^{6 \beta+3}}\right) \boldsymbol{\varepsilon}+\frac{2 \mu \beta}{\lambda^{6 \beta+3}} \mathbf{I} \operatorname{tr} \boldsymbol{\varepsilon}-p \mathbf{L} .
$$

Let us assume that the initial stresses $\boldsymbol{\tau}$ are small. Then we may neglect the last term in Eq. $(10)_{3}$. Thus, we obtain that

$$
\Theta=\mathcal{A} \cdot \varepsilon .
$$


From the symmetry consideration one can conclude that $\mathcal{A}$ has the structure of the elasticity tensor of the transversally isotropic solid with elastic constants depending on $\lambda, \lambda_{3}$ or $\tau, \tau_{3}$ and that $\boldsymbol{\Theta}=\boldsymbol{\Theta}^{\mathrm{T}}$. Note that $\mathcal{A}$ depends on the initial stressed state, i.e. $\mathcal{A}$ differs from the elastic tensor used in the linear elasticity, in general. In the special case of (12) the assumption (13) means that we neglected by the member $p\|\mathbf{L}\|$ and keep the dependence on $\lambda$ in other terms. Further we assume that (13) is invertible, i.e. $\boldsymbol{\varepsilon}=\mathcal{S} \cdots \Theta$ with $\mathcal{S}=\mathcal{A}^{-1}$. Thus, we establish that under the used assumptions the constitutive equations of the prestressed plate-like body are coincide with the Hooke's law for the transversally isotropic solids with elastic moduli depending on the initial stresses or the initial stretches.

Equation (13) allows to deduce the theory of plates made of prestressed material using the approach presented in [18-20,23,24]. Indeed, considering (13) as an analog of the Hooke's law one may obtain the effective stiffness tensors constructed for the plates made of linear orthotropic and transversally isotropic materials.

\section{$3 \quad 2 \mathrm{D}$ Plates equations}

Let us assume the geometrically and physically linear plate theory based on the so-called direct approach. In this case one states a two-dimensional deformable surface. On each part of this deformable surface forces and moments are acting - they are the primary variables. The next step is the introduction of the deformation measures. Finally, it is necessary to interlink the forces and the moments with the deformation variables (constitutive equations). Such a plate theory is formulated by a more natural way in comparison with the other approaches because it is so strong and so exact as the three-dimensional continuum mechanics formulation. But the identification of the stiffness and other parameters is a non-trivial problem and must be realized for each class of plates individually.

In the considered theory of plates we make two basic assumptions: 1) the plate (homogeneous or inhomogeneous in transverse direction) can be represented by a deformable surface $\mathcal{M}$, Fig. $2 ; 2$ ) each material point is an infinitesimal rigid body with 5 degrees of freedom (3 translations and 2 rotations).

In addition, the theory presented here is limited by small displacements and rotations and the quadratic strain energy density assumptions. The equilibrium 


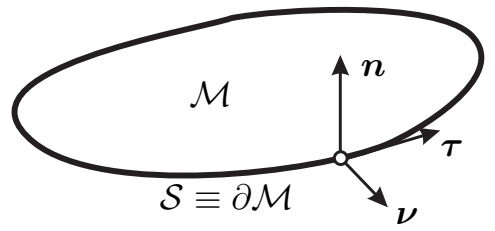

Fig. 2. Plate

equations and the kinematic equations are given by the relations [18,21-24]

$$
\begin{aligned}
& \nabla \cdot \mathbf{T}+\boldsymbol{q}=\mathbf{0}, \quad \nabla \cdot \mathbf{M}+\mathbf{T}_{\times}+\boldsymbol{m}=\mathbf{0}, \\
& \boldsymbol{\epsilon}=\frac{1}{2}\left[\nabla \boldsymbol{v}+(\nabla \boldsymbol{v})^{\mathrm{T}}\right], \quad \gamma=\nabla w-\boldsymbol{n} \times \boldsymbol{\varphi}, \quad \boldsymbol{\kappa}=\nabla \boldsymbol{\varphi} .
\end{aligned}
$$

Here $\mathbf{T}, \mathbf{M}$ are the tensors of forces and moments, $\boldsymbol{q}, \boldsymbol{m}$ are the surface loads (forces and moments), $\mathbf{T}_{\times}$is the vector invariant of the force tensor, $\nabla$ is the nabla operator, $\boldsymbol{v}=\boldsymbol{u} \cdot \mathbf{a}, w=\boldsymbol{u} \cdot \boldsymbol{n}, \boldsymbol{u}, \boldsymbol{\varphi}$ are the vectors of displacements and rotations. $\mathbf{a}$ is the first metric tensor, $\boldsymbol{n}$ is the unit normal vector, $\boldsymbol{\epsilon}, \boldsymbol{\gamma}$ and $\boldsymbol{\kappa}$ are the tensor of in-plane strains, the vector of transverse shear strains and the tensor of the out-of-plane strains, respectively.

The presented above plate kinematics is consistent with the following approximation of the vector of displacements of a three-dimensional elastic plate-like body

$$
\boldsymbol{w}(x, y, z)=\boldsymbol{u}(x, y)-z \boldsymbol{\varphi}(x, y)
$$

with kinematically independent fields of $\boldsymbol{u}$ and $\boldsymbol{\varphi}$. The surface strain energy density of a prestressed plate may be calculated from the relation

$$
U=\frac{1}{2}\langle\Theta \cdots \varepsilon\rangle
$$

where $\langle\ldots\rangle$ is the integral over the plate thickness $h$. The analogous approximation is used to construct the Reissner-Mindlin-type plate theory, see [3739]. The transition to the Kirchhoff-Love-type plate and shell theories may be performed using the dependence $\boldsymbol{\varphi}$ on $\boldsymbol{u}$, see [30,33] for details.

Within the direct approach in the case of an orthotropic material behavior and a plane mid-surface we assume the following surface strain energy and constitutive equations

$$
\begin{aligned}
U(\boldsymbol{\epsilon}, \boldsymbol{\gamma}, \boldsymbol{\kappa}) & =\frac{1}{2} \boldsymbol{\epsilon} \cdot \mathbf{A} \cdot \boldsymbol{\epsilon}+\boldsymbol{\epsilon} \cdot \mathbf{B} \cdot \boldsymbol{\kappa}+\frac{1}{2} \boldsymbol{\kappa} \cdot \mathbf{C} \cdot \boldsymbol{\kappa}+\frac{1}{2} \boldsymbol{\gamma} \cdot \boldsymbol{\Gamma} \cdot \boldsymbol{\gamma} \\
\mathbf{N} & \equiv \mathbf{T} \cdot \mathbf{a}=\frac{\partial U}{\partial \boldsymbol{\epsilon}}, \quad \boldsymbol{Q} \equiv \mathbf{T} \cdot n=\frac{\partial U}{\partial \boldsymbol{\gamma}}, \quad \mathbf{M}^{\mathrm{T}}=\frac{\partial U}{\partial \boldsymbol{\kappa}}
\end{aligned}
$$

$\mathbf{A}, \mathbf{B}, \mathbf{C}$ are $4^{\text {th }}$-order tensors, $\boldsymbol{\Gamma}$ is a $2^{\text {nd }}$-order tensor expressing the effective 
stiffness properties. They depend on the material properties and the crosssection geometry and are given by the relations [23]

$$
\begin{aligned}
& \mathbf{A}=A_{11} \mathbf{a}_{1} \mathbf{a}_{1}+A_{12}\left(\mathbf{a}_{1} \mathbf{a}_{2}+\mathbf{a}_{2} \mathbf{a}_{1}\right)+A_{22} \mathbf{a}_{2} \mathbf{a}_{2}+A_{44} \mathbf{a}_{4} \mathbf{a}_{4}, \\
& \mathbf{B}=B_{13} \mathbf{a}_{1} \mathbf{a}_{3}+B_{14} \mathbf{a}_{1} \mathbf{a}_{4}+B_{23} \mathbf{a}_{2} \mathbf{a}_{3}+B_{24} \mathbf{a}_{2} \mathbf{a}_{4}+B_{42} \mathbf{a}_{4} \mathbf{a}_{2}, \\
& \mathbf{C}=C_{22} \mathbf{a}_{2} \mathbf{a}_{2}+C_{33} \mathbf{a}_{3} \mathbf{a}_{3}+C_{34}\left(\mathbf{a}_{3} \mathbf{a}_{4}+\mathbf{a}_{4} \mathbf{a}_{3}\right)+C_{44} \mathbf{a}_{4} \mathbf{a}_{4}, \\
& \boldsymbol{\Gamma}=\Gamma_{1} \mathbf{a}_{1}+\Gamma_{2} \mathbf{a}_{2},
\end{aligned}
$$

where

$$
\mathbf{a}_{1}=\mathbf{a}=\boldsymbol{e}_{1} \boldsymbol{e}_{1}+\boldsymbol{e}_{2} \boldsymbol{e}_{2}, \mathbf{a}_{2}=\boldsymbol{e}_{1} \boldsymbol{e}_{1}-\boldsymbol{e}_{2} \boldsymbol{e}_{2}, \mathbf{a}_{3}=\boldsymbol{e}_{1} \boldsymbol{e}_{2}-\boldsymbol{e}_{2} \boldsymbol{e}_{1}, \mathbf{a}_{4}=\boldsymbol{e}_{1} \boldsymbol{e}_{2}+\boldsymbol{e}_{2} \boldsymbol{e}_{1}
$$

and $\boldsymbol{e}_{1}, \boldsymbol{e}_{2}$ are unit basis vectors of an orthonormal coordinate system. In addition, one obtains the orthogonality condition for $\mathbf{a}_{i}(i=1,2,3,4)$

$$
\frac{1}{2} \mathbf{a}_{i} \cdot \mathbf{a}_{j}=\delta_{i j}
$$

The structure of $U$ in (17) may be deduced from the consideration of (16) with the approximation (15).

The identification of the effective stiffness tensors $\mathbf{A}, \mathbf{B}, \mathbf{C}$ and $\boldsymbol{\Gamma}$ should be performed on the base of the properties of the real material. Let us assume the Hooke's law with material properties which depend on the normal coordinate $z$. The identification of the effective properties can be performed with the help of static boundary value problems (two-dimensional, three-dimensional) and the comparison of the forces and moments (in the sense of averaged stresses or stress resultants). Finally, we get the following expressions for the classical stiffness tensor components $[19,20,24]$

$$
\begin{aligned}
\left(A_{11} ;-B_{13} ; C_{33}\right) & =\frac{1}{4}\left\langle\frac{E_{1}+E_{2}+2 E_{1} \nu_{21}}{1-\nu_{12} \nu_{21}}\left(1 ; z ; z^{2}\right)\right\rangle, \\
\left(A_{22} ; B_{24} ; C_{44}\right) & =\frac{1}{4}\left\langle\frac{E_{1}+E_{2}-2 E_{1} \nu_{21}}{1-\nu_{12} \nu_{21}}\left(1 ; z ; z^{2}\right)\right\rangle, \\
\left(A_{12} ;-B_{23}=B_{14} ;-C_{34}\right) & =\frac{1}{4}\left\langle\frac{E_{1}-E_{2}}{1-\nu_{12} \nu_{21}} z\left(1 ; z ; z^{2}\right)\right\rangle, \\
\left(A_{44} ;-B_{42} ; C_{22}\right) & \left.=<G_{12}\left(1 ; z ; z^{2}\right)\right\rangle,
\end{aligned}
$$

where $E_{1}, E_{2}, \nu_{12}, \nu_{21}, G_{12}$ are the elastic moduli of the orthotropic bulk material. In addition, two non-classical stiffness terms are obtained

$$
\Gamma_{1}=\frac{1}{2}\left(\lambda^{2}+\eta^{2}\right) \frac{A_{44} C_{22}-B_{42}^{2}}{A_{44}}, \quad \Gamma_{2}=\frac{1}{2}\left(\eta^{2}-\lambda^{2}\right) \frac{A_{44} C_{22}-B_{42}^{2}}{A_{44}} .
$$


Here $\eta^{2}$ and $\lambda^{2}$ are the smallest non-zero eigen-values of Sturm-Liouville problems

$$
\frac{\mathrm{d}}{\mathrm{d} z}\left(G_{1 n} \frac{\mathrm{d} Z}{\mathrm{~d} z}\right)+\eta^{2} G_{12} Z=0, \quad \frac{\mathrm{d}}{\mathrm{d} z}\left(G_{2 n} \frac{\mathrm{d} Z}{\mathrm{~d} z}\right)+\lambda^{2} G_{12} Z=0
$$

with the boundary conditions

$$
\left.\frac{\mathrm{d} Z}{\mathrm{~d} z}\right|_{|z|=\frac{h}{2}}=0
$$

Let us remind that here the role of Hooke's law plays Eq. (13) with the tensor elasticity $\mathcal{A}$. If the bulk material is transversally isotropic then the stiffness tensors reduce to $[18]$

$$
\begin{aligned}
& \mathbf{A}=A_{11} \mathbf{a}_{1} \mathbf{a}_{1}+A_{22}\left(\mathbf{a}_{2} \mathbf{a}_{2}+\mathbf{a}_{4} \mathbf{a}_{4}\right), \\
& \mathbf{B}=B_{13} \mathbf{a}_{1} \mathbf{a}_{3}+B_{24}\left(\mathbf{a}_{2} \mathbf{a}_{4}-\mathbf{a}_{4} \mathbf{a}_{2}\right), \\
& \mathbf{C}=C_{22}\left(\mathbf{a}_{2} \mathbf{a}_{2}+\mathbf{a}_{4} \mathbf{a}_{4}\right)+C_{33} \mathbf{a}_{3} \mathbf{a}_{3}, \\
& \boldsymbol{\Gamma}=\Gamma_{1} \mathbf{a} .
\end{aligned}
$$

Here the $A_{11}, A_{22}, B_{13}, B_{24}, C_{22}, C_{33}$ and $\Gamma_{1}$ are given by the relations (19), (20) together with relations $E_{1}=E_{2}, \nu_{12}=\nu_{21}, \nu_{23}=\nu_{32}, G_{1 n}=G_{2 n}$, etc. Note that here these engineering constants are the components of the elasticity tensor $\mathcal{A}$ and depend on the initial stresses, in general.

The boundary conditions are given by

$$
\boldsymbol{\nu} \cdot \mathbf{T}=\boldsymbol{f}, \quad \boldsymbol{\nu} \cdot \mathrm{M}=\boldsymbol{l}, \quad(\boldsymbol{l} \cdot \boldsymbol{n}=0) \quad \text { along } \mathcal{S}_{f},
$$

and

$$
\boldsymbol{u}=\boldsymbol{u}^{0}, \boldsymbol{\varphi}=\varphi^{0} \quad \text { along } \mathcal{S}_{u}
$$

Here $\boldsymbol{f}$ and $\boldsymbol{l}$ are external force and couple vectors acting along the part $S_{f}$ of the boundary of the plate $\mathcal{S}=\mathcal{S}_{f} \cup \mathcal{S}_{u} \equiv \partial \mathcal{M}$, while $\boldsymbol{u}^{0}$ and $\varphi^{0}$ are given functions describing the displacements and rotation of the plate boundary $\mathcal{S}_{u}$, respectively. $\boldsymbol{\nu}$ is the unit normal vector to $\mathcal{S}(\boldsymbol{\nu} \cdot \boldsymbol{n}=0)$. The relations (21) and (22) are the static and kinematic boundary conditions, respectively. Other mixed types of boundary conditions are possible. For example, the simple support boundary conditions corresponding to a hinge are given by

$$
\nu \cdot \mathrm{M} \cdot \boldsymbol{t}=\mathbf{0}, \quad \boldsymbol{u}=\mathbf{0}, \quad \boldsymbol{\varphi} \cdot \boldsymbol{t}=0
$$

Here $\boldsymbol{t}$ is the unit tangent vector to $\mathcal{S}(\boldsymbol{t} \cdot \boldsymbol{n}=\boldsymbol{t} \cdot \boldsymbol{\nu}=0)$. 


\section{Conclusions}

Here we discussed the identification procedure of the components of the effective stiffness tensors in the linear theory of prestressed plates. The identification procedure bases on the comparison of solutions of few test problems for three-dimensional elastic solids and two-dimensional elastic plates. For the linear elastic behavior it was developed in $[19,20,24]$. Here we extend this approach to the case of an elastic material with initial stresses. Using some assumptions on the initial stress distribution we reduced the linearized equilibrium equations for the prestressed plate-like body to equations of a non-homogeneous transversally isotropic solid with elastic moduli depending on the initial stresses or stretches. Further we applied this approach and obtained the expressions for the components of the effective stiffness tensors. Within the described above approach the in-plane stiffness components, the bending stiffness components and the shear stiffness depend on the initial stresses.

Let us note there is at least one alternative way of deducing the theory of plates made of prestressed material which is free on the assumption of smallness of $\boldsymbol{\tau}$. One may use the linear in $z$ approximation $\boldsymbol{w}(x, y, z)=\boldsymbol{u}(x, y)-z \boldsymbol{\varphi}(x, y)$ to obtain the two-dimensional governing equations of a plate taking into account the initial stresses as well as the transverse shear deformations. Within this approach one obtains the presented here constitutive equations but for the determination of the shear stiffness some additional considerations may be needed.

The effective stiffness tensors are given in the general form for orthotropic material. All stiffness values are the result of averaging over the thickness of the plate-like body $h$. Only the last two transverse shear stiffness values should be estimated by solving a Sturm-Liouville problem. Assuming special three-dimensional material laws the values can be computed for any usual elastic or a prestressed body. In addition, since the material properties are assumed to be a function of the coordinate $z$ in the thickness direction the introduced formulaes can be applied even in the case of inhomogeneous in the thickness direction materials. Examples of such materials are laminated structures, sandwiches, but also foams and functionally graded materials.

\section{Acknowledgements}

The second author was supported by the DAAD program "Forschungsaufenthalte für Hochschullehrer und Wissenschaftler" in 2008. 


\section{References}

[1] S. Lee, N. Ramesh (Eds.), Polymeric Foams. Mechanisms and Materials, CRC Press, Boca Raton, 2004

[2] A. H. Landrock (Ed.), Handbook of Plastic foams. Types, Properties, Manufactore and Applications, Noes Publications, Park Ridge, New Jersey, 1995.

[3] L. J. Gibson, M. F. Ashby, Cellular Solids: Structure and Properties, 2nd Edition, Cambridge Solid State Science Series, Cambridge University Press, Cambridge, 1997.

[4] N. Mills, Polymer Foams Handbook. Engineering and Biomechanics Applications and Design Guide, Butterworth-Heinemann, Amsterdam, 2007.

[5] W. W. Feng, R. M. Christensen, Nonlinear deformation of elastomeric foams, International Journal of Non-Linear Mechanics 17 (5/6) (1982) 353-367.

[6] W. E. Warren, A. M. Kraynik, C. M. Stone, A constitutive model for twodimensional nonlinear elastic foams, Journal of the Mechanics and Physics of Solids 37 (6) (1989) 717-733.

[7] S. Demiray, W. Becker, J. Hohe, Analysis of two- and three-dimensional hyperelastic model foams under complex loading conditions, Mechanics of Materials 38 (2006) 985-1000.

[8] N. J. Mills, The high strain mechanical response of the wet Kelvin model for open-cell foams, International Journal of Solids and Structures 44 (2007) 51-65.

[9] N. J. Mills, R. Stämpfli, F. Marone, P. A. Brühwiler, Finite element micromechanics model of impact compression of closed-cell polymer foams, International Journal of Solids and Structures 46 (2009) 677-697.

[10] S. Jemiolo, S. Turteltaub, A parametric model for a class of foam-like isotropic hyperelastic materials, Transactions of the ASME. Journal of Applied Mechanics 67 (2000) 248-254.

[11] R. W. Ogden, Large deformation isotropic elasticity - on the correlation of theory and experiment for incompressible rubberlike solids, Proc. Roy. Soc. London A 326 (1972) 565-584.

[12] R. W. Ogden, Volume changes associated with the deformation of rubber-like solids, Journal of the Mechanics and Physics of Solids 24 (1976) 323-338.

[13] R. W. Ogden, Non-Linear Elastic Deformations, Dover Publications, New York, 1997.

[14] Y. Shen, F. Golnaraghi, A. Plumtree, Modelling compressive cyclic stressstrain behaviour of structural foam, International Journal of Fatigue 23 (2001) 491497. 
[15] R. Verdejo, N. J. Mills, Heelshoe interactions and the durabilityof EVA foam running-shoe midsoles, Journal of Biomechanics 37 (2004) 1379-1386.

[16] M. Schrodt, G. Benderoth, A. Kühhorn, G. Silber, Hyperelastic description of polymer soft foams at finite deformations, Technische Mechanik 25 (3-4) (2005) 162-173.

[17] B. Markert, A biphasic continuum approach for viscoelastic high-porosity foams: Comprehensive theory, numerics, and application, Archive of Computational Methods in Engineering 15 (2008) 371-446.

[18] H. Altenbach, P. A. Zhilin, A general theory of elastic simple shells (in Russ.), Uspekhi Mekhaniki 11 (4) (1988) 107-148.

[19] H. Altenbach, An alternative determination of transverse shear stiffnesses for sandwich and laminated plates, International Journal of Solids and Structures 37 (25) (2000) 3503-3520.

[20] H. Altenbach, On the determination of transverse shear stiffnesses of orthotropic plates, Zeitschrift für Angewandte Mathematik und Physik 51 (2000) 629-649.

[21] H. Altenbach, P. A. Zhilin, The theory of simple elastic shells, in: R. Kienzler, H. Altenbach, I. Ott (Eds.), Critical review of the theories of plates and shells and new applications, Lecture Notes Applied Computational Mechanics 16, Springer, Berlin, 2004, pp. 1-12.

[22] P. A. Zhilin, Mechanics of deformable directed surfaces, International Journal of Solids and Structures 12 (1976) $635-648$.

[23] P. A. Zhilin, Applied Mechanics. Foundations of the Theory of Shells (in Russ.), St. Petersburg State Polytechnical University, 2006.

[24] H. Altenbach, V. A. Eremeyev, Direct approach based analysis of plates composed of functionally graded materials, Archive of Applied Mechanics 78 (10) (2008) 775-794.

[25] H. Altenbach, V. A. Eremeyev, Analysis of the viscoelastic behavior of plates made of functionally graded materials, Zeitschrift für angewandte Mathematik und Mechanik 88 (5) (2007) 332-341.

[26] H. Altenbach, V. A. Eremeyev, On the bending of viscoelastic plates made of polymer foams, Acta Mechanica 204 (3-4) (2009) 137-154.

[27] H. Altenbach, V. A. Eremeyev, Thin-walled structures made of foams, in: H. Altenbach and A. Öchsner (Ed.), Cellular and Porous Materials: Modeling Testing - Application, CISM Courses and Lecture Notes No. 521, Springer, Wien, 2010, pp. 167-242.

[28] D. Ieşan, Prestressed Bodies, Vol. 195 of Pitman Research Notes in Mathematics Series, Longman Scientific and Technical, London, 1989.

[29] A. I. Lurie, Nonlinear Theory of Elasticity, North-Holland, Amsterdam, 1990. 
[30] A. E. Green, W. Zerna, Theoretical Elasticity, 2nd Edition, Dover Publications, New York, 1992.

[31] A. I. Lurie, Theory of Elasticity, Foundations of Engineering Mechanics, Springer, Berlin, 2005.

[32] Y. B. Fu, R. W. Ogden, Nonlinear stability analysis of pre-stressed elastic bodies, Continuum Mechanics and Thermodynamics 11 (1999) 141-172.

[33] L. M. Zubov, Theory of small deformations of prestressed thin shells, Journal of Applied Mathematics and Mechanics 40 (1) (1976) 73-82.

[34] D. M. Haughton, R. W. Ogden, On the incremental equations in non-linear elasticity-I. Membrane theory, Journal of the Mechanics and Physics of Solids 26 (1978) 93-110.

[35] D. M. Haughton, R. W. Ogden, On the incremental equations in nonlinear elasticity-II. Bifurcation of pressurized spherical shells, Journal of the Mechanics and Physics of Solids 26 (1978) 111-138.

[36] L. M. Zubov, Compatibility equations, stress functions, and variational principles in the theory of prestressed shells, Journal of Applied Mathematics and Mechanics 49 (1) (1985) 95-100.

[37] E. Reissner, The effect of transverse shear deformation on the bending of elastic plates, Journal of Applied Mechanics 12 (11) (1945) A69 - A77.

[38] E. Reissner, On bending of elastic plates, Quarterly of Applied Mathamatics 5 (1947) 55-68.

[39] R. D. Mindlin, Influence of rotatory inertia and shear on flexural motions of isotropic elastic plates, Transaction ASME. Journal of Applied Mechanics 18 (1951) 31-38.

[40] E. I. Grigolyuk, I. T. Selezov, Nonclassical theories of vibration of beams, plates and shells (in Russ.), in: Itogi nauki i tekhniki, Vol. 5 of Mekhanika tverdogo deformiruemogo tela, VINITI, Moskva, 1973. 